\title{
Mutational analysis of an archaeal minichromosome maintenance protein exterior hairpin reveals critical residues for helicase activity and DNA binding
}

Aaron S Brewster ${ }^{\dagger}$, Ian M Slaymaker ${ }^{\dagger}$, Samir A Afif, Xiaojiang S Chen ${ }^{*}$

\begin{abstract}
Background: The mini-chromosome maintenance protein (MCM) complex is an essential replicative helicase for DNA replication in Archaea and Eukaryotes. While the eukaryotic complex consists of six homologous proteins (MCM2-7), the archaeon Sulfolobus solfataricus has only one MCM protein (ssoMCM), six subunits of which form a homohexamer. We have recently reported a $4.35 \AA$ crystal structure of the near full-length SsoMCM. The structure reveals a total of four $\beta$-hairpins per subunit, three of which are located within the main channel or side channels of the ssoMCM hexamer model generated based on the symmetry of the N-terminal Methanothermobacter thermautotrophicus (mtMCM) structure. The fourth $\beta$-hairpin, however, is located on the exterior of the hexamer, near the exit of the putative side channels and next to the ATP binding pocket.

Results: In order to better understand this hairpin's role in DNA binding and helicase activity, we performed a detailed mutational and biochemical analysis of nine residues on this exterior $\beta$-hairpin (EXT-hp). We examined the activities of the mutants related to their helicase function, including hexamerization, ATPase, DNA binding and helicase activities. The assays showed that some of the residues on this EXT-hp play a role for DNA binding as well as for helicase activity.

Conclusions: These results implicate several current theories regarding helicase activity by this critical hexameric enzyme. As the data suggest that EXT-hp is involved in DNA binding, the results reported here imply that the EXThp located near the exterior exit of the side channels may play a role in contacting DNA substrate in a manner that affects DNA unwinding.
\end{abstract}

\section{Background}

DNA replication is a tightly regulated and efficient process. Central to this process is the coordinated unwinding of double stranded DNA by the AAA+ family member MCM (Minichromosome Maintenance) protein [1-3]. As the replicative helicase, $\bar{M} C M$ is required for cellular viability, and is regulated through controlled assembly at the origin in combination with ORC and Cdc6 among others, and through phosphorylation by various replication checkpoint proteins such as CDK and DDK [4-6].

\footnotetext{
* Correspondence: xiaojiang.chen@usc.edu

† Contributed equally

Molecular and Computational Biology, University of Southern California, Los Angeles, CA 90089 USA
}

In eukaryotes, $\mathrm{MCM}$ is composed of a heterohexamer formed from the gene products of 6 homologs (MCM27), all necessary for cell survival [5,7]. Archaeal MCM serves as a model system for studying MCM function as many strains only have one MCM gene whose product oligomerizes as a homohexamer or even as a double hexamer [8-10]. Several structures have been recently made available that have helped understanding of the biochemistry involved in DNA unwinding (reviewed in [11-13]. Specifically, the poorly-conserved N-terminal portion was solved in a double hexameric configuration from Methanothermobacter thermautotrophicus (N-mtMCM) [10], and as single hexamers from Sulfolobus solfataricus (N-ssoMCM) [14]. The near full length MCM monomer from Sulfolobus solfataricus (ssoMCM) was also recently 
solved [15]. Finally, the structure of an inactive MCM homolog with natural internal deletions from Methanopyrus kandleri (mkaMCM2) was also published [16].

The crystal structure of ssoMCM reveals an elongated fold for the monomer with two large domains. The first is a large $\mathrm{N}$-terminal domain with its sequence, but not structure, poorly conserved. The second is a highly conserved C-terminal helicase domain that contains what is known as the MCM box [15]. The hexamer structures, and hexamer models of the near-full length structures, reveal a large central channel, through which DNA is postulated to be threaded.

One of the major structural features of the subunit structure of ssoMCM is the four obvious $\beta$-hairpins projecting radially away from the monomeric ssoMCM. One, located in the N-terminal domain (NT-hairpin), projects into the central channel and has been implicated in DNA binding $[10,17]$. The other three $\beta$-hairpins are located in the $\mathrm{C}$-terminal $\mathrm{AAA}+$ domain: the pre-sensor 1 hairpin (PS1-hp), the helix 2 insertion hairpin (H2I-hp), and the external hairpin (EXT-hp) [15].

Residues on the PS1-hp and H2I-hp play a role for helicase activity, are involved in DNA binding and project into or near the central channel $[17,18]$. The EXT$\mathrm{hp}$, however, is located on the exterior side of the hexamer, near the side channels in the C-terminal domain [15]. The unusual location of this EXT-hp raises interesting questions regarding its functional role. However, unlike the other $\beta$-hairpins, no detailed mutational and functional analysis of the EXT-hp has been performed, and its role in DNA binding, ATPase, and helicase activity is not understood. In this work we present a granular examination of this important structural feature, including a thorough examination of the hairpin's role in DNA binding, ATP hydrolysis and helicase activity.

\section{Results}

\section{Mutational analysis of the exterior hairpin}

The EXT-hp sequence is semi-conserved in archaea and eukaryotes (Figure 1A), raising intriguing questions as to the functional roles of this hairpin next to the side channel on the exterior of the hexamer. Preliminary analysis suggested the hairpin may be involved in unwinding [15]. However, it is unclear which specific residues of the hairpin are involved in helicase function and in what manner they facilitate activity. To understand this hairpin's role in helicase function, we created a series of 10 single and double alanine mutants (numbered M1 to M10). The DNA binding, ATPase and Helicase activities were then examined for each mutant. Nine of the ten mutants are on the EXT-hp; one control mutant is on the Walker A lysine involved in ATP binding and hydrolysis (Figure 1B). The nine hairpin mutants are located in three regions, an inside $\beta$-strand that faces towards the interior side of the hexamer and side channel, a tip, and an outside $\beta$-strand that faces away from hexamer (Figures 1C, D).

Mutant M1 modifies a highly conserved lysine to alanine on the exterior side of the hairpin, close to the hairpin base. Mutants M2-M7 are located in the VLED sequence that wraps around the tip of the hairpin and consist of single or double mutations. We previously postulated that these residues could function to repel DNA, similar to the acidic pin in RuvA [15,19]. M2-M4 change the properties of the hydrophobic residues, VL. Of the two, the leucine is more conserved but neither are strongly conserved in eukaryotes or archaea. M5-M8 mutate the acidic ED region. Of note, the aspartate is highly conserved in archaea, but in eukaryotes its conservation varies by homolog. For example, in MCM5 it remains an aspartate, but in MCM3 it is consistently changed to asparagine. M8 changes a well conserved arginine in archaea that only remains consistently basic in MCM7, and mostly so in MCM2. M9, changing an absolutely conserved arginine at the base of the hairpin, had been assayed previously for ATPase and helicase activity [20], and was included as an additional control. We further assayed it for DNA binding differentials. Finally, M10, the Walker A mutant, changes the conserved lysine vital for ATP hydrolysis [9]. The Walker A motif is not conserved in the inactive MCM homolog from Methanopyrus kandleri [16], nor is the EXT-hp present in that homolog.

\section{Oligomerization as assayed by size-exclusion chromatography}

We first assayed oligomeric state to determine if these mutations would affect folding or hexamerization compared with wild type. At $250 \mathrm{mM} \mathrm{NaCl}$, wild type ssoMCM eluted on a Superose 6 size exclusion column as a hexamer. At $1 \mathrm{M} \mathrm{NaCl}$, it eluted at a lower oligomeric state that is likely a dimer or monomer. We found previously that mutations that affect hexamerization can either cause the elution peak to shift to a lower oligomeric species, or can cause it to elute as two peaks, a hexameric peak and one at a lower oligomeric state, as if it was ran at $1 \mathrm{M} \mathrm{NaCl}$ [15]. As shown in Figure $2 \mathrm{~A}$, none of the 10 mutants reported here eluted as a split peak in gel filtration, and most eluted predominantly as a hexamer at $250 \mathrm{mM} \mathrm{NaCl}$. While mutants M5 and M8 eluted slighter faster than WT and mutants M1 and M4 eluted slightly slower, their peak positions remained near the $440 \mathrm{kD}$ marker (WT MCM hexamer is about $460 \mathrm{kD}$ ). Similar minor variations in elution profiles have been observed previously for a panel of ssoMCM mutants [20]. Thus it seems that none of the mutants have obvious defects in oligomerization, though mutants M1, M4, M5 and M8 may be slightly affected. 


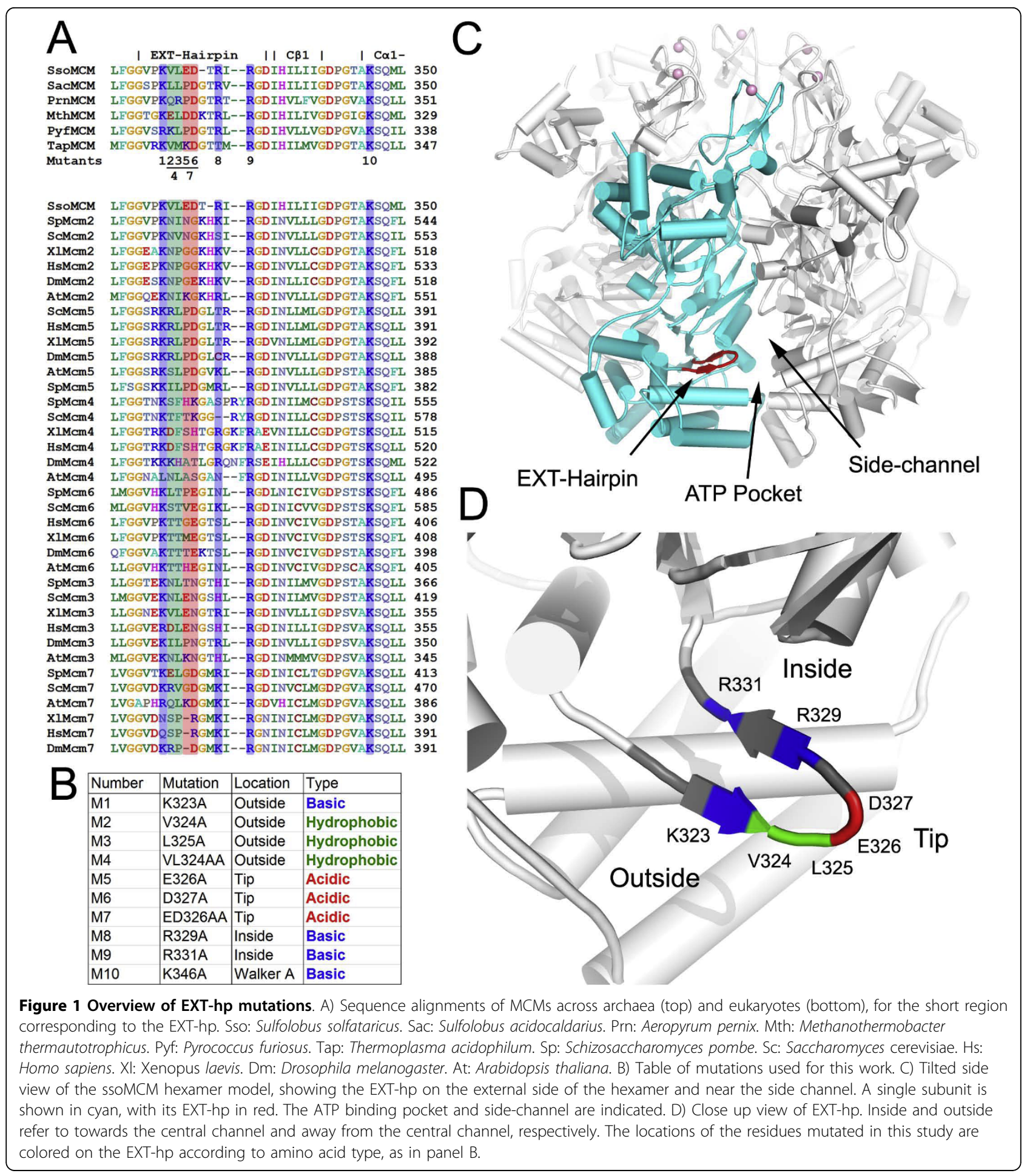

\section{ATPase activity}

We next performed a series of biochemical assays of the mutants to examine their effect on ATP hydrolysis and other activities. Protein concentrations for these assays were calibrated by nano-drop and further confirmed by SDS-PAGE in Figure 2B. The first set of assays determined $k_{\text {cat }}$ and $k_{\mathrm{m}}$ values for ATP hydrolysis via the Enzchek phosphate release assay, as described previously [15]. Activity curves for wild-type and mutants M3 and M8 are shown in Figure 2C. For each experiment, an additional test was performed to measure ATPase stimulation upon addition of Y-shaped fork 


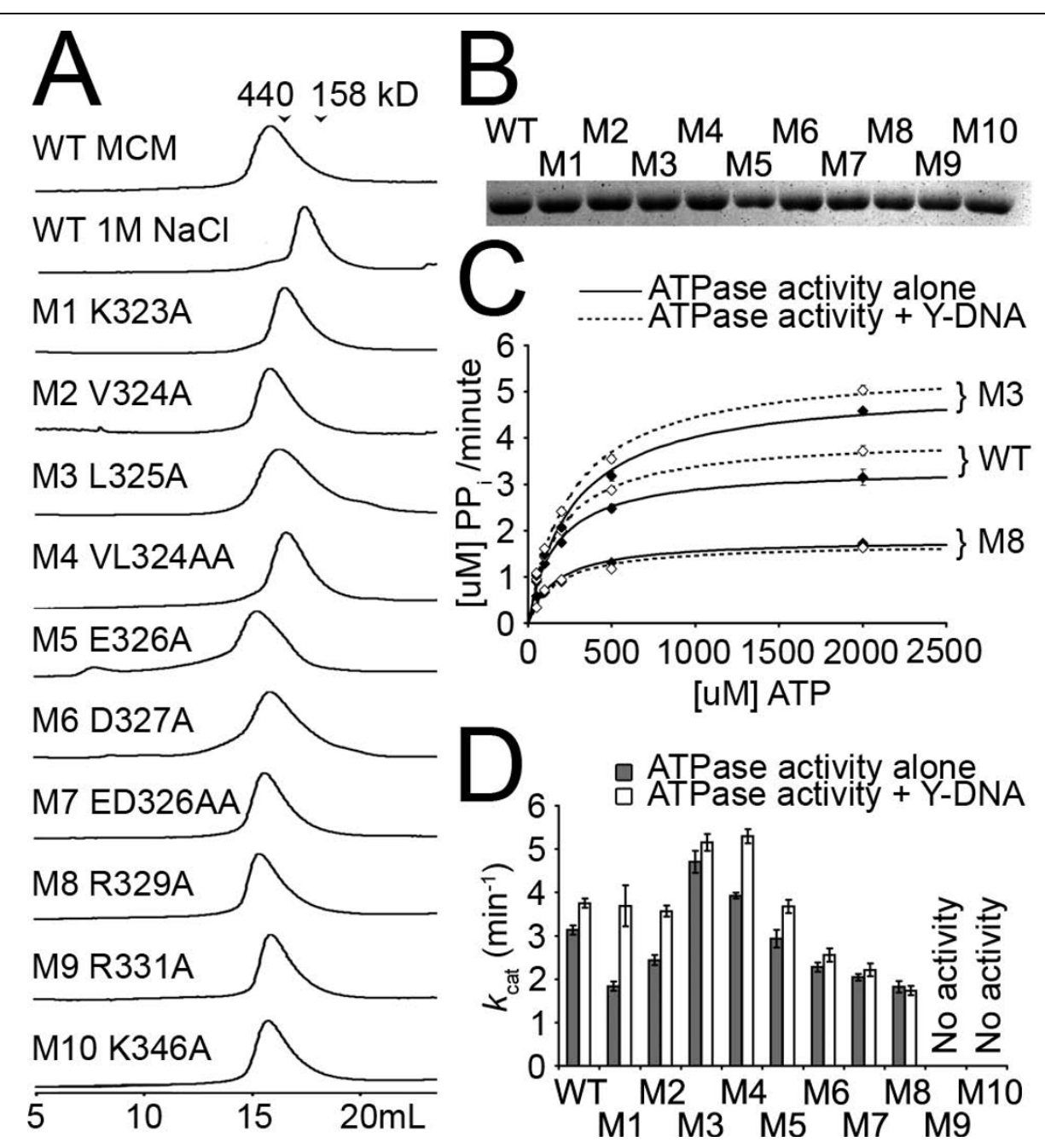

Figure 2 The results of FPLC and ATPase analysis. A) FPLC analysis of the mutations by gel filtration chromatography on a Superose- 6 column. Molecular marker positions, Ferritin $(440 \mathrm{kD})$ and aldolase $(158 \mathrm{kD})$, are indicated by arrows. B) SDS-PAGE gel analysis of the purified mutant proteins. C) ATPase activity curves for WT, M3 and M8 in the presence and absence of Y-shaped DNA. Error bars representing standard error of the mean are present, but in most cases are too small to see. D) Summary of ATPase data in the presence (black bar) and absence (white bar) of Y-shaped DNA. Error bars are standard error from curve fitting.

DNA. WT shows a modest stimulation by the Y-shaped DNA, as noted previously. M3's ATPase activity is significantly higher than WT's. M8's ATPase activity is lower, and furthers loses ATPase stimulation upon the addition of DNA. A summary of $k_{\text {cat }}$ changes, together with the full data, including $\mathrm{Km}$ values, is given in Figure $2 \mathrm{D}$ and listed in Table 1.

\section{DNA-binding activity}

DNA-binding activity of the mutants was determined by EMSAs using a single stranded and forked DNA substrate (Figure 3, binding constants in Table 1). Generally speaking, mutations of the hydrophobic tip (mutants M2-M4) increased DNA binding slightly. Mutations in the conserved aspartate on the tip of the hairpin (M6) showed a large decrease in dsDNA binding, and the double acidic mutant (M7) had a similar decrease in ssDNA and dsDNA binding. All of the basic mutations, including $\mathrm{M} 1$ on the outside of the hairpin, decreased ssDNA binding somewhat, and dsDNA binding significantly, with M8, R329A having the largest effect. These findings indicate that this hairpin is involved in DNA binding. Additionally, we see highly cooperative DNA binding in our assays. Previously, using fluorescence polarization anisotropy, we had seen Hill factors for YDNA binding ranging from 1.3 to 2.5 [15], suggesting cooperativity of DNA binding. Now, using EMSA assays, we see Hill factors ranging from 4 to as high as 15 for both ssDNA and Y-DNA, confirming cooperativity. The cooperative binding is likely related to the 
Table 1 Kinetic parameters of EXT-hp mutants

\begin{tabular}{|c|c|c|c|c|c|c|c|c|}
\hline \multirow[b]{2}{*}{ Mutant } & \multicolumn{2}{|c|}{ ATpase activity } & \multicolumn{2}{|c|}{ ATpase + Y-DNA } & \multicolumn{2}{|c|}{ ssDNA Binding } & \multicolumn{2}{|c|}{ Y-DNA Binding } \\
\hline & $k_{\text {cat }, \min ^{-1}}$ & $k_{\mathrm{m},}, \mathrm{nM}$ & $k_{\text {cat }}, \min ^{-1}$ & $k_{\mathrm{m},}, \mathrm{nM}$ & $k_{d}, n M$ & Hill & $k_{d}, n M$ & Hill \\
\hline$\overline{W T}$ & $3.1 \pm 0.1$ & $170 \pm 20$ & $3.8 \pm 0.1$ & $190 \pm 20$ & $240 \pm 0.5$ & $8.8 \pm 0.5$ & $224 \pm 7.3$ & $5.8 \pm 1.3$ \\
\hline M1 & $1.8 \pm 0.1$ & $140 \pm 30$ & $3.7 \pm 0.5$ & $440 \pm 150$ & $317 \pm 5.8$ & $4.4 \pm 0.3$ & $398 \pm 4.0$ & $13.2 \pm 1.3$ \\
\hline M2 & $2.4 \pm 0.1$ & $120 \pm 20$ & $3.6 \pm 0.1$ & $160 \pm 20$ & $209 \pm 5.8$ & $4.7 \pm 0.5$ & $230 \pm 7.2$ & $10.1 \pm 3.3$ \\
\hline M3 & $4.7 \pm 0.3$ & $270 \pm 40$ & $5.2 \pm 0.2$ & $260 \pm 30$ & $169 \pm 1.3$ & $5.6 \pm 0.1$ & $186 \pm 6.4$ & $7.9 \pm 1.1$ \\
\hline M4 & $3.9 \pm 0.1$ & $230 \pm 10$ & $5.3 \pm 0.2$ & $260 \pm 30$ & $165 \pm 10.0$ & $13.4 \pm 4.3$ & $178 \pm 1.5$ & $7.7 \pm 0.5$ \\
\hline M5 & $2.9 \pm 0.2$ & $60 \pm 40$ & $3.7 \pm 0.2$ & $180 \pm 30$ & $185 \pm 0.3$ & $12.1 \pm 0.3$ & $224 \pm 0.9$ & $11.6 \pm 0.4$ \\
\hline M6 & $2.3 \pm 0.1$ & $100 \pm 20$ & $2.6 \pm 0.2$ & $130 \pm 30$ & $283 \pm 78.7$ & $15.6 \pm 20.1$ & $400 \pm 6.5$ & $5.6 \pm 0.4$ \\
\hline M7 & $2.0 \pm 0.1$ & $90 \pm 10$ & $2.2 \pm 0.1$ & $90 \pm 20$ & $444 \pm 1.0$ & $10.6 \pm 0.5$ & $404 \pm 2.6$ & $8.8 \pm 0.4$ \\
\hline M8 & $1.8 \pm 0.1$ & $170 \pm 40$ & $1.7 \pm 0.1$ & $170 \pm 30$ & $380 \pm 1.8$ & $8.0 \pm 0.3$ & $483 \pm 0.4$ & $9.0 \pm 0.03$ \\
\hline M9 & No Activity & & No Activity & & $325 \pm 8.3$ & $6.2 \pm 0.8$ & $428 \pm 2.0$ & $12.9 \pm 0.5$ \\
\hline M10 & No Activity & & No Activity & & $267 \pm 7.0$ & $4.5 \pm 0.5$ & $339 \pm 2.6$ & $11.4 \pm 2.0$ \\
\hline
\end{tabular}

All values are calculated based on monomeric MCM subunit. For each value, standard error is given. Hill: Hill cooperativity coefficient.

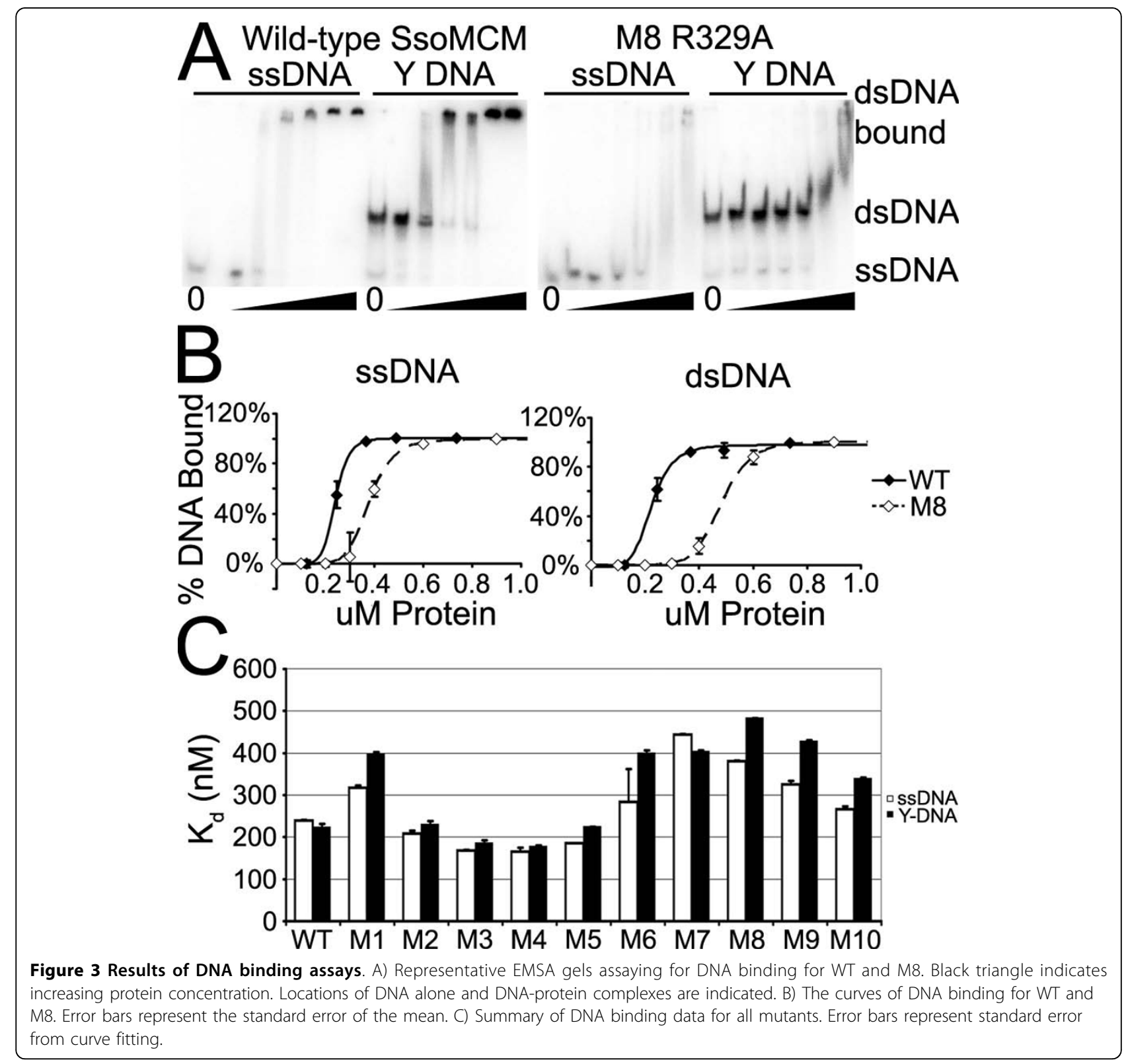


oligomerization of the six subunits of ssoMCM around the DNA. The higher Hill factors reported here could come from differences in experimental conditions; for example we have added a heating step by treating the protein-DNA mixture at $65^{\circ} \mathrm{C}$ for 30 minutes during sample preparation, which may allow the protein to bind DNA better.

\section{Helicase activity}

Helicase assays were then performed on these mutants using radio-labeled forked DNA substrates (Figure 4). As expected, the R331A and Walker A mutations, which had no detectable ATPase activity, exhibited no helicase activity, as shown previously $[9,20]$. Generally speaking, those mutants with lower ATPase and DNA binding activity also have lower helicase activity. However, the deficits in ATPase and DNA binding of some of the mutations did not strictly correlate with the level of deficits in helicase activity. In particular, the acidic mutations that showed significant decreases in DNA binding (M6 and M7), and decreases in ATPase activity, did not suffer significant decreases in helicase activity, at least under the assay conditions used here.

\section{Discussion}

In this report, we conduct a systematic mutational and functional study in order to understand how this externally located $\beta$-hairpin immediately next to the exit of the side channels is involved in helicase function. We

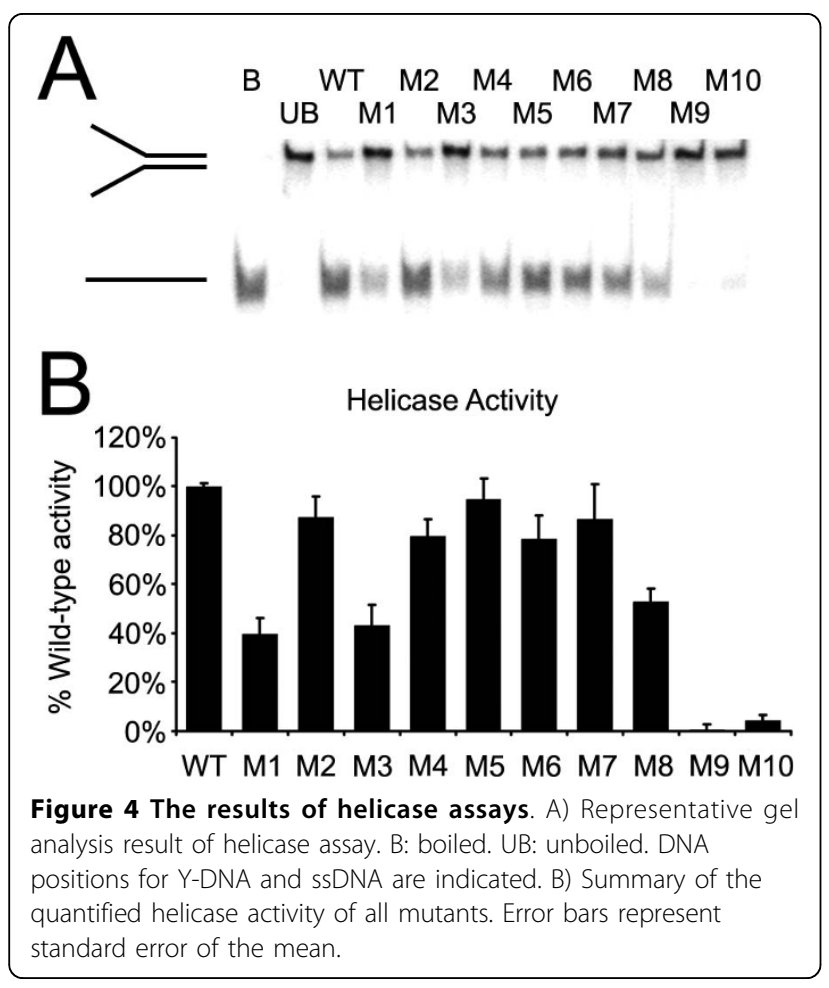

do this by assessing specific oligomerization, DNA binding, ATPase, and DNA unwinding functionality of the mutants compared with wild type MCM.

The EXT-hp contains residues with hydrophobic, acidic, and basic properties. The residues were mutated to alanine to examine the contributions of each of these residues to the various activities associated with helicase function. A summary of the data is listed in Table 2. Mutants M2-M4 contain mutations of hydrophobic residues. M2 weakens ATPase activity, but retains DNA binding and helicase activity. Its effect is minor. However, M3, located near the tip of the EXT-hp, is complex. It exhibits stronger ATPase activity than WT but its DNA induced stimulation of ATPase activity is lessened. It also has somewhat tighter DNA binding, particularly to ssDNA. Finally, its helicase activity is greatly compromised. We conclude that this mutant may slightly de-couple ATP activity from DNA unwinding. The double mutant M4 has slightly higher ATPase activity than WT but not as high as M3, and it retains DNA stimulation of the ATPase activity, DNA binding, and helicase activity.

Mutants M5-M7 contain mutations of acidic residues (Table 2). M5 showed a slight increase in ssDNA binding, but no change from WT in all other aspects. M6, the more highly conserved of the acidic residues, revealed weaker ATPase activity, Y-DNA binding and helicase activity. It is interesting that the loss of the aspartate decreased DNA binding. The puzzling data in this set, however, is the double mutant, M7. Despite the weaker ATPase activity and DNA binding, it showed near wild-type helicase activity.

Mutants involving basic residues (M1, M8-M9) have the most profound effect in all the activities assayed. Reduced ATPase activity, DNA binding and helicase activity are observed across the board. Notably, R331 (M9), which had been assayed for ATPase and helicase activity previously [20], now is revealed to have compromised DNA binding as well.

Our single and double mutant EMSA assays from the current round of experiments reveal decreases in DNA binding on the order of 1.5 to 2 fold increases in $\mathrm{K}_{\mathrm{d}}$. Mutations in the PS1-hairpin resulted in a similar change in DNA binding ( $2 \times$ fold increase in $K_{d}$ ) [17]. Therefore, the EXT-hp appears to be involved in interactions with DNA, as shown for the three other $\beta$-hairpin structural elements in the MCM structure.

Some of the specific changes in DNA binding caused by mutations appear to be counter intuitive. For example M6 and M7, which are both mutations eliminating negative charges, have a lowered affinity for DNA. Also, the increase in binding from hydrophobic mutations is interesting. Similarly, how two basic amino acids on opposite sides of the hairpin (K323 (M1) and R329 
Table 2 Summary of biochemical assays of the mutants

\begin{tabular}{|c|c|c|c|c|c|c|c|}
\hline \multirow[b]{2}{*}{ Mutant No. } & \multirow[b]{2}{*}{ Mutant } & \multirow[b]{2}{*}{ Oligomeric State } & \multicolumn{2}{|l|}{ ATPase } & \multicolumn{2}{|c|}{ DNA Binding } & \multirow[b]{2}{*}{ Helicase } \\
\hline & & & ATPase & Stimulation & ssDNA & Y-DNA & \\
\hline$\overline{\mathrm{WT}}$ & - & Hexamer & +++ & +++ & +++ & +++ & +++ \\
\hline $\mathrm{M} 2$ & V324A & Hexamer & ++ & +++ & +++ & +++ & +++ \\
\hline M3 & L325A & Hexamer & ++++ & ++ & ++++ & +++ & + \\
\hline M4 & VL324AA & Hexamer & +++ & +++ & ++++ & +++ & ++ \\
\hline M5 & E326A & Hexamer & +++ & +++ & ++++ & +++ & +++ \\
\hline M6 & D327A & Hexamer & ++ & ++ & +++ & + & ++ \\
\hline M7 & ED327AA & Hexamer & ++ & ++ & + & + & +++ \\
\hline$\overline{M 1}$ & R323A & Hexamer & ++ & +++ & ++ & + & + \\
\hline M8 & R329A & Hexamer & ++ & ++ & + & + & + \\
\hline M9 & R331A & Hexamer & 0 & 0 & + & + & 0 \\
\hline M10 & K346A & Hexamer & 0 & 0 & +++ & ++ & 0 \\
\hline
\end{tabular}

Mutations are grouped by residue type (M2-M4: hydrophobic, M5-M7: acidic, M1, M8-M10: basic). +++: wild type activity level. ++++: greater than wild type (more activity or tighter binding). ++: less than wild type. +: substantially less than wild type. 0 : no detectible activity. ATPase stimulation refers to the increase of ATPase activity upon addition of Y-DNA.

(M8)) could impact DNA binding in similar ways is unclear. Regardless of the individual peculiarities of the mutants, there seems to be a general trend when looking at the data as a whole. With the exception of mutant M1, the functional role of the residues generally increases in importance from the base of the hairpin to the tip, with the residues located in the internal $\beta$-strand of the EXT-hairpin being the most important for ATPase activity, DNA binding and helicase activity.

The proximity of R329 (M8) (decreased DNA binding, decreased helicase activity) to the putative side-channel could implicate the hairpin in pulling ssDNA through the channel in a side channel extrusion model (see [15]). However, additional explanations are feasible. One possibility is, as proposed by Rothenberg et. al. in a DNA exclusion model [21], that one ssDNA strand may be on the exterior of the hexamer interacting with the EXT-hp while the other ssDNA is translocated through the central channel.

However, it is important to note that decreases in DNA binding were often correlated with decreases in ATPase activity. Defects in ATP binding could prevent conformational changes necessary for DNA binding. Thus these DNA binding defects could be secondary effects. Alternatively, DNA binding stimulates ATPase activity, so mutations with DNA binding phenotypes could also result in slowing the rate of ATP hydrolysis as a secondary effect. This is at least partially borne out by the fact that the $k_{\mathrm{m}}$ 's for ATPase activity do not seem unduly affected by the mutations, with the exceptions of M9 and M10 (Table 1). Thus, the enzyme may be still binding ATP with wild-type affinity, but hydrolyzes ATP slower due to problems with DNA binding.

Finally, R331 (M9) is a residue of particular interest since we first examined the ssoMCM structure and compared it to known mutations [20,22]. As part of an examination of sequence alignments and structure alignments of ssoMCM with viral hexameric helicase LargeT antigen (LTag), we discovered that R331 aligns well with LTag K418 that serves as a "lysine finger" and, in combination with LTag's arginine finger, coordinates the ATP gamma phosphate in the ATP binding and hydrolysis [22-24]. K418 is vital for LTag ATPase and helicase activity [25], which mimics the phenotype we see here. Thus, the base of the EXT-hp seems directly involved in ATP hydrolysis.

\section{Conclusions}

We have presented for the first time the systematic mutational and biochemical analysis of the residues on the EXT-hp to understand their functional roles in MCM helicase activity. The study suggests that key residues on the EXT-hp affect helicase activity through their DNA binding activity. Determining how this EXThp interacts with DNA through these residues and how such interactions are related to unwinding activity will require further structural and functional analysis.

\section{Methods}

\section{Cloning, purification and size-exclusion analysis}

WT Sulfolobus solfataricus MCM and mutations were cloned and purified in wash buffer (WB: $50 \mathrm{mM}$ Tris-Cl (pH 8.0), $250 \mathrm{mM} \mathrm{NaCl}, 1 \mathrm{mM}$ DTT), as described previously [15] with the following changes: prior to sizeexclusion chromatography, the protein was first purified on a $6 \mathrm{~mL}$ Resource Q anion exchange column. Protein concentration was assayed by nano-drop and SDSPAGE analysis. The protein was then diluted to $10 \mathrm{uM}$ in helicase buffer $(\mathrm{HB}, 30 \mathrm{mM}$ Tris acetate $(\mathrm{pH} 8)$, $75 \mathrm{mM} \mathrm{NaCl}, 50 \mathrm{mM}$ potassium acetate, $10 \mathrm{mM}$ 
magnesium acetate), aliquoted, and frozen at $-80^{\circ} \mathrm{C} . \sim 0.5$ $\mathrm{mg}$ of protein was taken separately to analyze on sizeexclusion chromatography.

\section{Helicase assays}

Helicase assays were performed exactly as described previously on radio-labeled Y-shaped DNA substrate [15]. The substrate was created from annealing two complementary strands. The sequences are: $(\mathrm{dT})_{44}$ GCTCG TGCAGACGTCGAGGTGAGGACGAGCTCCTCGTGACCACG (strand Y1) and CGTGGTCACGAGGAGCTCGTCCTCACCTCGACGTCTGCACGAGC $(\mathrm{dT})_{44}$ (strand Y2). Experiments were performed in triplicate.

\section{Electrophoretic Mobility Shift Assays}

DNA binding constants were determined using electrophoretic mobility shift assays (EMSAs). ssDNA (strand Y2) or Y-shaped DNA from above was radio labeled and desalted using a Micro Bio-Spin 6 Column (BIO-RAD). Increasing amounts of protein were incubated with 1.4 nM DNA in DNA binding buffer (DB, $20 \mathrm{mM}$ Tris-Cl (pH 7.5), $100 \mathrm{mM} \mathrm{NaCl}, 2 \mathrm{mM}$ EDTA, $0.5 \mathrm{mM}$ magnesium chloride) for 30 minutes at $65^{\circ} \mathrm{C}$, then ran on a $5 \%$ polyacrylamide gel in $0.5 \times$ Tris/borate/EDTA buffer for 90 minutes at $100 \mathrm{~V}$. DNA bands were detected by autoradiography and quantified. \% DNA bound was determined vs. protein concentration and $K_{d}$ was calculated as described in [25]. Experiments were performed in duplicate.

\section{ATPase assays}

ATPase assays were performed as described previously [15]. Experiments were performed in triplicate.

\section{Acknowledgements}

This work is supported by NIH grant GM080338.

\section{Authors' contributions}

$A B$ designed and performed the experiments, including helicase and EMSA assays, and wrote the manuscript. IS made the clones, assisted in protein purification and performed the ATPase assays. SA assisted in protein purification and performed the size exclusion analysis. XC participated in the study's design and coordination and revised the manuscript. All authors read and approved the manuscript.

Received: 13 April 2010 Accepted: 18 August 2010 Published: 18 August 2010

\section{References}

1. Lee JK, Hurwitz J: Processive DNA helicase activity of the minichromosome maintenance proteins 4,6 , and 7 complex requires forked DNA structures. Proc Natl Acad Sci USA 2001, 98(1):54-59.

2. Ishimi Y: A DNA helicase activity is associated with an MCM4, -6 , and -7 protein complex. J Biol Chem 1997, 272(39):24508-24513.

3. You Z, Komamura $Y$, Ishimi Y: Biochemical analysis of the intrinsic Mcm4Mcm6-mcm7 DNA helicase activity. Mol Cell Biol 1999, 19(12):8003-8015.

4. Mendez J, Stillman B: Perpetuating the double helix: molecular machines at eukaryotic DNA replication origins. Bioessays 2003, 25(12):1158-1167.
5. Bell SP, Dutta A: DNA replication in eukaryotic cells. Annu Rev Biochem 2002, 71:333-374.

6. Bowers JL, Randell JC, Chen S, Bell SP: ATP hydrolysis by ORC catalyzes reiterative $\mathrm{Mcm} 2-7$ assembly at a defined origin of replication. Mol Cell 2004, 16(6):967-978

7. Tye BK: MCM proteins in DNA replication. Annu Rev Biochem 1999, 68:649-686.

8. Kelman Z, Lee JK, Hurwitz J: The single minichromosome maintenance protein of Methanobacterium thermoautotrophicum DeltaH contains DNA helicase activity. Proc Natl Acad Sci USA 1999, 96(26):14783-14788.

9. Chong JP, Hayashi MK, Simon MN, XU RM, Stillman B: A double-hexamer archaeal minichromosome maintenance protein is an ATP-dependent DNA helicase. Proc Natl Acad Sci USA 2000, 97(4):1530-1535.

10. Fletcher RJ, Bishop BE, Leon RP, Sclafani RA, Ogata CM, Chen XS: The structure and function of MCM from archaeal M. Thermoautotrophicum. Nat Struct Biol 2003, 10(3):160-167.

11. Sakakibara N, Kelman LM, Kelman Z: Unwinding the structure and function of the archaeal MCM helicase. Mol Microbiol 2009, 72(2):286-296.

12. Bochman ML, Schwacha A: The mcm complex: unwinding the mechanism of a replicative helicase. Microbiol Mol Biol Rev 2009 73(4):652-683

13. Costa A, Onesti S: Structural biology of MCM helicases. Crit Rev Biochem Mol Biol 2009, 44(5):326-342.

14. Liu W, Pucci B, Rossi M, Pisani FM, Ladenstein R: Structural analysis of the Sulfolobus solfataricus MCM protein N-terminal domain. Nucleic Acids Res 2008, 36(10):3235-3243

15. Brewster AS, Wang G, Yu X, Greenleaf WB, Carazo JM, Tjajadia M, Klein MG, Chen XS: Crystal structure of a near-full-length archaeal MCM: functional insights for an AAA+ hexameric helicase. Proc Natl Acad Sci USA 2008, 105(51):20191-20196.

16. Bae B, Chen YH, Costa A, Onesti S, Brunzelle JS, Lin Y, Cann IK, Nair SK: Insights into the architecture of the replicative helicase from the structure of an archaeal MCM homolog. Structure 2009, 17(2):211-222.

17. McGeoch AT, Trakselis MA, Laskey RA, Bell SD: Organization of the archaeal MCM complex on DNA and implications for the helicase mechanism. Nat Struct Mol Biol 2005, 12(9):756-762.

18. Jenkinson ER, Chong JPJ: Minichromosome maintenance helicase activity is controlled by $\mathrm{N}$ - and C-terminal motifs and requires the ATPase domain helix-2 insert. Proc Natl Acad Sci USA 2006, 103(20):7613-7618.

19. Ingleston SM, Sharples GJ, Lloyd RG: The acidic pin of RuvA modulates Holliday junction binding and processing by the RuvABC resolvasome. EMBO J 2000, 19(22):6266-6274.

20. Moreau MJ, McGeoch AT, Lowe AR, Itzhaki LS, Bell SD: ATPase site architecture and helicase mechanism of an archaeal MCM. Mol Cell 2007, 28(2):304-314.

21. Rothenberg E, Trakselis MA, Bell SD, Ha T: MCM forked substrate specificity involves dynamic interaction with the 5'-tail. J Biol Chem 2007, 282(47):34229-34234

22. Brewster AS, Chen XS: Insights into the MCM functional mechanism: lessons learned from the archaeal MCM complex. Crit Rev Biochem Mol Biol 2010, 45(3):243-256.

23. Li D, Zhao R, Lilyestrom W, Gai D, Zhang R, DeCaprio JA, Fanning E, Jochimiak A, Szakonyi G, Chen XS: Structure of the replicative helicase of the oncoprotein SV40 large tumour antigen. Nature 2003, 423(6939):512-518

24. Gai D, Zhao R, Li D, Finkielstein CV, Chen XS: Mechanisms of conformational change for a replicative hexameric helicase of SV40 large tumor antigen. Cell 2004, 119(1):47-60.

25. Greenleaf WB, Shen J, Gai D, Chen XS: Systematic study of the functions for the residues around the nucleotide pocket in simian virus $40 \mathrm{AAA}+$ hexameric helicase. J Virol 2008, 82(12):6017-6023.

doi:10.1186/1471-2199-11-62

Cite this article as: Brewster et al:: Mutational analysis of an archaeal minichromosome maintenance protein exterior hairpin reveals critical residues for helicase activity and DNA binding. BMC Molecular Biology 2010 11:62. 